\title{
Prediction of DNA-binding protein based on statistical and geometric features and support vector machines
}

\author{
Weiqiang Zhou ${ }^{1 *}$, Hong Yan ${ }^{1,2}$ \\ From International Workshop on Computational Proteomics \\ Hong Kong, China. 18-21 December 2010
}

\begin{abstract}
Background: Previous studies on protein-DNA interaction mostly focused on the bound structure of DNA-binding proteins but few paid enough attention to the unbound structures. As more new proteins are discovered, it is useful and imperative to develop algorithms for the functional prediction of unbound proteins. In our work, we apply an alpha shape model to represent the surface structure of the protein-DNA complex and extract useful statistical and geometric features, and use structural alignment and support vector machines for the prediction of unbound DNA-binding proteins.

Results: The performance of our method is evaluated by discriminating a set of 104 DNA-binding proteins from 401 non-DNA-binding proteins. In the same test, the proposed method outperforms the other method using conditional probability. The results achieved by our proposed method for; precision, 83.33\%; accuracy, 86.53\%; and MCC, 0.5368 demonstrate its good performance.

Conclusions: In this study we develop an effective method for the prediction of protein-DNA interactions based on statistical and geometric features and support vector machines. Our results show that interface surface features play an important role in protein-DNA interaction. Our technique is able to predict unbound DNA-binding protein and discriminatory DNA-binding proteins from proteins that bind with other molecules.
\end{abstract}

\section{Background}

More and more structural data are becoming available for biomolecules, which provide valuable resources for the study of biomolecular interactions. In recent years scientists have made a lot of efforts in studying proteinDNA interactions based on X-ray crystallography and NMR data. Samudrala and Moult proposed an all-atom distance-dependent discriminatory function for the prediction of nucleic acid binding proteins [1]. Later, Moont et al. applied an interface pairwise residue level potential to the screening of predicted docked complex [2]. Recently, Robertson and Varani improved the method based an interface-atom distance-dependent

\footnotetext{
* Correspondence: kenandzhou@hotmail.com

'Department of Electronic Engineering, City University of Hong Kong, Kowloon, Hong Kong

Full list of author information is available at the end of the article
}

formalism and showed that this obtained better prediction power than previous methods [3]. However, these studies mainly focussed on the bound structure of DNA-binding protein. The ability to recognize DNAbinding sites in structures that are unbound is potentially useful. There are several other methods that have been devised to predict protein-related interactions: Lo et al. used residue contacts to study the helix-helix interaction in membrane proteins [4], Xu et al. applied collective matrix factorization in the prediction of protein-protein interaction [5], Gonzalez et al. introduced correlation analysis based method in the prediction of protein-ligand interaction [6], Ahmad proposed the usage of moment information in the prediction of DNA-binding proteins [7].

Previous attempts to predict protein-DNA interaction provided acceptable results, but the underlying principle 
of protein-DNA interactions is not fully understood. As the progress made in genomic projects, more high resolution 3D structures of biological molecules have become available now. However, few of the previous studies paid enough attention to the $3 \mathrm{D}$ interface surface characteristics of the protein-DNA complex. Interface surface characteristics such as atom type, residue type, surface curvature, accessible surface area, etc. play important roles in protein-DNA interaction. In order to analysis the properties of the molecules, a 3D model is need. Alpha shape has been proved to be useful in molecule analysis and has been used for in molecular volume computation, cavities detection and shape representation. Liang et al. first proposed to use alpha shape modeling to compute the molecular area, volume and to detect the inaccessible cavities in proteins $[8,9]$. Li et al. used the edges in alpha shape modeling to represent the protein structure and atom contacts [10]. Poupon used Voronoi tessellations to compute the protein volume and detect the pockets, cavities and voids on the protein surface [11]. Recently, alpha shape has been introduced into the study of molecular surfaces. Albou et al. applied alpha shape modeling to characterize the surface of the proteins and defined the surface residue and surface patches [12]. Zhou and Yan applied alpha shape modeling and conditional probability in the study of proteinDNA interface properties [13,14].

In this, we propose to apply $3 \mathrm{D}$ alpha shape modeling to study the interface surface characteristics of the protein-DNA complex and develop an algorithm for the prediction of unbound DNA-binding proteins based on statistical and geometric features and support vector machine.

\section{Methods}

\section{Data selection}

Trainset: This training dataset contains 199 DNA-binding proteins with different functions constructed by
Zhou and Yan [13] to serve as the correct structure data. The control data contains 86 RNA-binding proteins, 106 ligand-binding proteins [13] and 186 proteinbinding proteins [15].

Testset: The testing dataset contains 104 unbound DNA-binding proteins and 401 non-DNA-binding proteins as per Zhou and Yan [13].

TemLib: The correct protein-DNA complex dataset contains the same 199 different types of DNA-binding proteins used in Trainset which form the template library for structural alignment.

\section{Alpha shape modeling}

Alpha shape modeling has been used to study the molecular structures such as the detection of pockets in known structures, computation of the molecular volume and description of the protein surface $[11,12]$. In this study, we apply the 3D alpha shape model to the reconstruction of the interface of protein-DNA interaction and used geometric features to represent the properties of protein-DNA interaction.

We can construct the 3D alpha shape based on the Delaunay triangulation [16]. The alpha shape is a subset of the tetrahedrons in the Delaunay triangulation complex which is a generalization of the convex hall of the point set [17] (the atoms in a molecule). Comparison between the edges of the Delaunay triangulation of a protein-DNA complex and the edges of the alpha shape is shown in Figure 1. We can see from Figure 1(B) that the alpha shape can be created by trimming the edges of the Delaunay triangulation.

Theoretically, the alpha shape can be computed as follows. First, we define the alpha complex based on a set of points $\{P\}$. Given the value of $\alpha$, the alpha complex includes all the simplexes in the Delaunay triangulation which have an empty circumsphere ('empty' means that the open sphere does not include any points of $\{P\})$ with a squared radius equal to, or smaller than, $\alpha$. The alpha
A

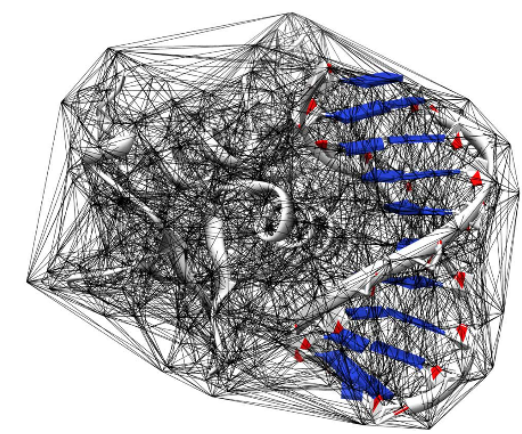

B

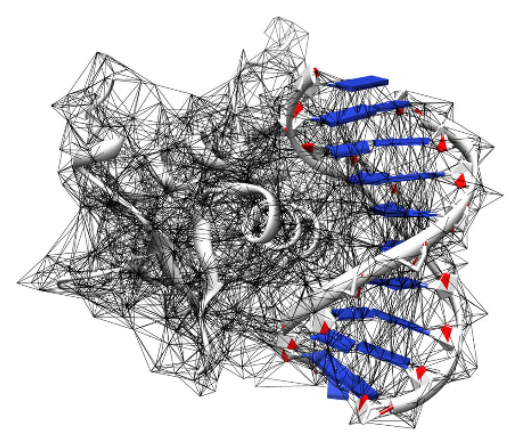

Figure 1 Alpha shape construction (A) The edges of the Delaunay triangulation of a protein-DNA complex. (B) The edges of the alpha shape obtained from the Delaunay triangulation. 
shape can be obtained by the domain covered by the simplexes of the alpha complex. Notice that, the alpha value here actually controls the roughness of the molecular surface obtained. In this work, we use the CGAL [18] library to compute the alpha shape.

An important step before extracting geometric features from the alpha shape model is the definition of interface surface which contains most of the information need to predict protein-DNA interaction. In the alpha shape model, the vertices correspond to the surface atoms of the original protein-DNA structure. Using this characteristic, the interface atoms of the protein-DNA structure can be defined as follows. First, we calculate the alpha shape of the protein-DNA complex and obtain the surface atom set $\{$ Complex\}. Then, we calculate the alpha shape of the protein independently (with the DNA part removed) and obtain the protein surface atom set \{Protein\}. Finally, the interface atoms set can be obtained by retaining the atoms which are in $\{$ Protein $\}$ but not in $\{$ Complex\}.

\section{Structural alignment}

In order to predict the potential interface between the unbound proteins and DNA, we make use of structural alignment. For a target protein structure, for which we would like to predict whether it will bind to any DNA, we carry out structure alignment against the template library (TemLib) using TM-align [19]. The target structure is scanned against every structure in the template library to find the most similar structure as defined by the largest TM-scored [19]. In Testset, any template with a sequence overlap larger than $35 \%$ with another template is excluded from the template library to avoid the over-training problem. After structural alignment, a new protein-DNA structure is created by replacing the selected template sequence with the aligned target protein structure (as shown in Figure 2).

\section{Statistical and features}

\section{Residue Index}

Considering the specificity of the interface residue types, we define a feature-named residue index by computing the percentage of each residue type showing up in the interface for each interface atom set defined above. All 20 residue types are considered and a residue index is represented as a vector in the following format: (ALA\%, ARG\%, ASN\%, ASP\%, CYS\%, GLU\%, GLN, GLY\%, HIS \%, ILE\%, LEU\%, LYS\%, MET\%, PHE\%, PRO\%, SER\%, THR\%, TRP\%, TYR\%, VAL\%).

\section{TM-score}

The TM-score is obtained using TM-align [19] in the structure alignment process. It is a measure of the similarity between the template protein structure and the target protein structure.

\section{Curvature Index}

In order to characterize the interface properties geometrically, we calculate the interface surface curvature. It is derived from the solid angle $\Omega$ of the interface atoms in the alpha shape model, and it is transformed to the range of -1 (cleft) to 1 (knob) using $\cos (\Omega / 4)$. The curvature index is defined as a vector-containing convexand concave index: (Convex Index, Concave Index). The convex index represents the overall knob level of the interface which is a summation of the positive solid angles in the interface, and it is normalized by the number of interface atoms. The concave index, on the other hand, represents the overall cleft level of the interface which is a summation of the negative solid angles, and it is also normalized by the number of interface atoms.

\section{Mean Connectivity}

The connectivity of the surface atoms in the molecule is introduced by Zhou and Yan [20]. The vertices of the alpha shape model correspond to the surface atoms of the molecule being reconstructed. The connectivity of a surface atom is defined as the connection between this


Figure 2 Structural alignment process (A) The template DNA sequence with its interacting protein structure. (B) The new structure formed by the target protein structure and the template DNA sequence. 
atom and the other surface atoms, which correspond to the edges in the alpha shape model. Mean connectivity is computed by summing up the connectivity of all the interface atoms and normalized by the number of interface atoms.

\section{Interface Atom Index}

The interface atom index is defined by the percentage of the interface atom taken in the overall protein-protein complex, i.e., the ratio of the number of interface atoms to the number of atoms in the whole complex. It is a measure of the interface size relative to the complex size.

\section{Support vector machine (SVM)}

SVM has been widely used in pattern classification problems. As a machine learning algorithm, SVM has to build the classification rules based on the existing knowledge. This type of learning method requires training data with known class labels. The input data of the SVM are vectors. In this sense, SVM is suitable for high dimensional data classification. In this study, we use the mySVM software [21] to predict protein-DNA interaction based on the statistical and geometric features.

\section{Performance evaluation}

In order to evaluate the performance of the prediction result, we use different measures including Recall, Specificity, Precision, Accuracy and Matthews correlation coefficient (MCC). First, we define TP (true positive, the number of proteins correctly predicted as DNA-binding), FP (false positive, the number of proteins incorrectly predicted as DNA-binding), TN (true negative, the number of proteins correctly predicted as nonDNA-binding) and FN (false negative, the number of proteins incorrectly predicted as non-DNA-binding). The definitions of the measures are as follows:

Recall: $T P /(T P+F N)$;

Specificity: $T N /(T N+F P)$;

Precision: $T P /(T P+F P)$;

Accuracy: $(T P+T N) /(T P+F N+T N+F P)$;

MCC: $((T P \times T N)-(F P \times F N)) / \sqrt{(T P+F P) \times(T P+F N) \times(T N+F P) \times(T N+F N)}$.

\section{Results}

In order to evaluate the performance of the proposed method, we perform two experiments to discriminate the unbound DNA-binding proteins from the unbound non-DNA-binding proteins in Testset using the proposed method and the discriminatory function developed by Zhou and Yan [13] respectively.

\section{Performance of SVM classifier}

Structural alignment is carried out using TemLib as the template library for the Trainset and Testset. Then we construct the alpha shape model of the new structures based on CGAL [18]. The alpha value is set to be the value to obtain one connected component automatically in CGAL. Features are extracted from the potential DNA-binding interface surface information of the proteins. A vector of 25 dimensions is used to represent each structure in the following format: \{residue index, TM-score, curvature index, mean connectivity, interface atom index\}.

The pattern recognition SVM is used with the radial kernel implemented in mySVM. When the parameters $C, \gamma$ and elision+ are set to $0.1,0.01$ and 0.5 respectively [21], mySVM produced a good classification performance for both sensitivity and specificity. The label for the correct data is set to 1 and the label for the control data is set to -1 for SVM training. Performance of the classification for Testset is shown in Table 1. Within 104 unbound DNA-binding proteins, 54 proteins are correctly recognized which results in a percentage accuracy for Recall of 43.27. All the other performance evaluation measures reach a very high value due to 392 in 401 unbound non-DNA-binding proteins being correctly recognized. The overall performance is; Precision, 83.33\%; Accuracy, 86.53\%; and MCC 0.5368. These results demonstrate that the proposed method shows good performance in the classification of non-DNAbinding proteins from the DNA-binding proteins.

\section{Performance comparison with the conditional probability discriminatory function}

In the conditional probability discriminatory function developed by Zhou and Yan [13], three features are considered: atom type, residue type and surface curvature. They used the conditional probability method to construct a scoring function for the potential DNA-binding proteins. To be consistent with the results in [13], we use the same training data and choose 200 for the number of bins and 14 for the alpha value as mentioned in their work. (The related data set and program are obtained from http://www.hy8.com/bioinformatics.htm).

According to their method, the best classification can be obtained by setting the threshold rage from -1.7 to -2.3 . We use -1.7 and -2.3 to compute the performance evaluation measure mentioned in the Methods section. The result is shown in Table 1.

Table 1 Comparison of performance between the
conditional probability discriminatory function (CPDF)
and SVM classifier (SC).
\begin{tabular}{cccccc}
\hline Method & Recall & Specificity & Precision & Accuracy & MCC \\
\hline CPDF (-1.7) & $48.08 \%$ & $70.57 \%$ & $29.76 \%$ & $65.94 \%$ & 0.1601 \\
CPDF (-2.3) & $44.23 \%$ & $82.29 \%$ & $39.32 \%$ & $74.46 \%$ & 0.2542 \\
SC & $43.27 \%$ & $97.76 \%$ & $83.33 \%$ & $86.53 \%$ & 0.5368 \\
\hline
\end{tabular}




\section{Discussion}

Comparing the results of the two methods, we can see that both methods show good performance in recognizing DNA-binding proteins. The conditional probability discriminatory function (CPDF) shows better performance in recognizing the DNA-binding proteins than the SVM classifier (SC) in terms of Recall. However, in terms of Specificity and Precision, SC outperforms CPDF in the discrimination of non-DNA-binding proteins. The reason is that the method developed in this study considers a wider range of interface surface features. This result shows that interface surface features are dramatically different between DNA-binding proteins and non-DNA-binding proteins. Accuracy and MCC measures the overall performance of the classifier, we can see that due to the low false positive rate these two measures of SC are much better than that of CPDF. From this comparison, we can see that the method developed in this study shows comparable ability in recognizing the DNA-binding proteins with the previous method [13] and outperforms the previous method in recognizing non-DNA-binding proteins.

\section{Conclusions}

In this paper, we propose a method based on structural alignment to predict protein-DNA interaction. TM-align is used to search for proper template structures in structural alignment. New structures are made by replacing the original protein structures with the target proteins. We apply a 3D alpha shape model to represent the interface of the protein-DNA complex, and we extract statistical and geometric features including residue index, TM-score, curvature index, mean connectivity and an interface atom index to characterize the interface properties. The SVM is used to classify DNA-binding proteins and non-DNA-binding proteins using the interface features. The performance of our method is tested by discriminating 104 DNA-binding protein structures from 401 non-DNA-binding proteins. We use different evaluation measures to represent the experiment output of our method. In the experiment, we have achieved an accuracy of $86.53 \%$ and an MCC of 0.5368 . Our method outperforms the conditional probability discriminatory function in terms of MCC, which indicates the good performance achieved by our method in discriminating between DNA-binding proteins and non-DNA-binding proteins. This study shows that interface surface features play an important role in protein-DNA interaction. Our method is able to predict unbound DNA-binding proteins and discriminatory DNA-binding proteins from proteins that bind with other molecules. The result indicates the potential usage of the proposed method in the study of protein and other related fields.

\section{Acknowledgements}

This work is supported by the Hong Kong Research Grant Council (Project CityU 123809) and City University of Hong Kong (Project 7008094). This article has been published as part of Proteome Science Volume 9 Supplement 1, 2011: Proceedings of the International Workshop on Computational Proteomics. The full contents of the supplement are available online at http://www.proteomesci.com/supplements/9/S1.

\section{Author details}

'Department of Electronic Engineering, City University of Hong Kong, Kowloon, Hong Kong. ${ }^{2}$ School of Electrical and Information Engineering, University of Sydney, NSW2006, Australia.

\section{Authors' contributions}

WQ carried out the programming and analysis work. HY initiated the project and provided suggestions on the formulations based on alpha shapes. Both authors read and approved the final manuscript.

\section{Competing interests}

The author(s) declare that they have no competing interests.

Published: 14 October 2011

\section{References}

1. Samudrala R, Moult J: An all-atom distance-dependent conditional probability discriminatory function for protein structure prediction. $J \mathrm{Mo} /$ Biol 1998, 275:895-916.

2. Moont G, Gabb HA, Sternberg MJE: Use of pair potentials across protein interfaces in screening predicted docked complexes. Proteins-Structure Function and Genetics 1999, 35:364-373.

3. Robertson TA, Varani G: An all-atom, distance-dependent scoring function for the prediction of protein-DNA interactions from structure. Proteins 2007, 66:359-374.

4. Lo A, Chiu YY, R dland EA, Lyu PC, Sung TY, Hsu WL: Predicting helix-helix interactions from residue contacts in membrane proteins. Bioinformatics 2009, 25:996.

5. $\mathrm{Xu} Q, \mathrm{~W} . \mathrm{XE}$, Yang Q: Protein-protein interaction prediction via Collective Matrix Factorization. 2010 IEEE International Conference on Bioinformatics and Biomedicine (BIBM), ; 18-21 Dec. 2010 2010, 62-67.

6. Gonzalez AJ, Li L, Wu CH: Predicting ligand binding residues using multipositional correlations and kernel canonical correlation analysis. Bioinformatics and Biomedicine (BIBM), 2010 IEEE International Conference on; 18-21 Dec. 2010 2010, 158-163.

7. Ahmad S, Sarai A: Moment-based prediction of DNA-binding proteins. J Mol Biol 2004, 341:65-71.

8. Liang J, Edelsbrunner H, Fu P, Sudhakar PV, Subramaniam S: Analytical shape computation of macromolecules: I. Molecular area and volume through alpha shape. Proteins 1998, 33:1-17.

9. Liang J, Edelsbrunner H, Fu P, Sudhakar PV, Subramaniam S: Analytical shape computation of macromolecules: II. Inaccessible cavities in proteins. Proteins 1998, 33:18-29.

10. Li $X, H u C Y$, Liang J: Simplicial edge representation of protein structures and alpha contact potential with confidence measure. Proteins 2003, 53:792-805.

11. Poupon A: Voronoi and Voronoi-related tessellations in studies of protein structure and interaction. Curr Opin Struc Biol 2004, 14:233-241.

12. Albou LP, Schwarz B, Poch O, Wurtz JM, Moras D: Defining and characterizing protein surface using alpha shapes. Proteins 2009, 76:1-12.

13. Zhou W, Yan H: A Discriminatory Function for Prediction of Protein-DNA Interactions based on Alpha Shape Modeling. Bioinformatics 2010, 26:2541-2548.

14. Zhou W, Yan H: Prediction of DNA-binding protein based on alpha shape modeling. 2010 IEEE International Conference on Bioinformatics and Biomedicine (BIBM), ; 18-21 Dec. 2010 2010, 23-28.

15. Murakami Y, Mizuguchi K: Applying the Naive Bayes classifier with kernel density estimation to the prediction of protein-protein interaction sites. Bioinformatics 2010, 26:1841-1848.

16. Delaunay B: Sur la sphère vide. Izvestia Akademii Nauk SSSR, Otdelenie Matematicheskii i Estestvennyka Nauk 1934, 7:793-800. 
17. Edelsbrunner H, Mucke EP: Three-Dimensional Alpha-Shapes. Acm T Graphic 1994, 13:43-72.

18. Computational Geometry Algorithms Library. [http://www.cgal.org].

19. Zhang $Y$, Skolnick J: TM-align: a protein structure alignment algorithm based on the TM-score. Nucleic Acids Res 2005, 33:2302-2309.

20. Zhou W, Yan H: Relationship between periodic dinucleotides and the nucleosome structure revealed by alpha shape modeling. Chemical Physics Letters 2010, 489:225-228.

21. Rüping S: mySVM-Manual. University of Dortmund, Lehrstuhl Informatik 8; 2000 [http://www-ai.cs.uni-dortmund.de/SOFTWARE/MYSVM/].

doi:10.1186/1477-5956-9-S1-S1

Cite this article as: Zhou and Yan: Prediction of DNA-binding protein based on statistical and geometric features and support vector machines. Proteome Science 2011 9(Suppl 1):S1.

Submit your next manuscript to BioMed Central and take full advantage of:

- Convenient online submission

- Thorough peer review

- No space constraints or color figure charges

- Immediate publication on acceptance

- Inclusion in PubMed, CAS, Scopus and Google Scholar

- Research which is freely available for redistribution

Submit your manuscript at www.biomedcentral.com/submit 